\title{
DETERMINANT INEQUALITIES FOR SIEVED ULTRASPHERICAL POLYNOMIALS
}

\section{J. BUSTOZ and I. S. PYUNG}

(Received 28 March 2000)

\begin{abstract}
Paul Turan first observed that the Legendre polynomials satisfy the inequality $P_{n}^{2}(x)-P_{n-1}(x) P_{n}(x)>0,-1<x<1$. Inequalities of this type have since been proved for both classical and nonclassical orthogonal polynomials. In this paper, we prove such an inequality for sieved orthogonal polynomials of the second kind.
\end{abstract}

2000 Mathematics Subject Classification. Primary 33C47; Secondary 33E30.

1. Introduction. It was observed by Paul Turán [9] that the Legendre polynomials satisfy the determinant inequality

$$
P_{n}^{2}(x)-P_{n+1}(x) P_{n-1}(x)>0, \quad-1<x<1, n=0,1, \ldots
$$

G. Szegö [8] gave two very beautiful proofs of Turán's inequality. In the years since Szegö's paper appeared, it has been proved by various authors $[5,6,7]$ that inequality (1.1) is satisfied by the classical orthogonal polynomials. In general, let $\left\{P_{n}(x)\right\}$ be a sequence of polynomials orthogonal in an interval $[a, b]$. Then the polynomials must satisfy a recursion

$$
P_{n+1}(x)=\left(A_{n} x+B_{n}\right) P_{n}(x)-C_{n} P_{n-1}(x), \quad n=0,1, \ldots,
$$

where we define $P_{-1}(x)=0$.

We begin with a very simple result shows that, inequalities of Turán type are satisfied by any sequence of orthogonal polynomials.

THEOREM 1.1. If the polynomials $\left\{P_{n}(x)\right\}$ are orthogonal on $a \leq x \leq b$, then for each $n$ there exists $c_{n}, a \leq c_{n} \leq b$, such that

$$
\frac{P_{n}^{2}(x)}{P_{n}^{2}\left(c_{n}\right)}-\frac{P_{n+1}(x)}{P_{n+1}\left(c_{n}\right)} \frac{P_{n-1}(x)}{P_{n-1}\left(c_{n}\right)} \geq 0, \quad a \leq x \leq b .
$$

Proof. Consider the quotient

$$
f_{n}(x)=\frac{P_{n+1}(x) P_{n-1}(x)}{P_{n}^{2}(x)} .
$$

Obviously, the roots of $P_{n}(x)$ are singularities and apart from these points $f_{n}(x)$ is continuous. Also, for each root $x_{k, n}$ of $P_{n}(x), k=1,2, \ldots, n$, there is an open interval $I_{n}$ centered at $x_{k n}$ in which $P_{n+1}(x) P_{n-1}(x)<0$. This follows from the recursion (1.2). 
Consequently, $f_{n}(x)$ is bounded above and must take on a positive maximum value at a point $c_{n} \in[a, b]$. Thus

$$
\frac{P_{n+1}\left(c_{n}\right) P_{n-1}\left(c_{n}\right)}{P_{n}^{2}\left(c_{n}\right)} \geq \frac{P_{n+1}(x) P_{n-1}(x)}{P_{n}^{2}(x)}, a \leq x \leq b .
$$

This proves the theorem.

Although Theorem 1.1 asserts that Turán's inequality is a simple consequence of orthogonality, it is generally quite difficult to determine the point $c_{n}$ referred to in Theorem 1.1. In the case of the classical orthogonal polynomials of Jacobi and their special case, the Gegenbauer polynomials, the point $c_{n}=1$ is an endpoint of the interval of orthogonality $[-1,1]$. Turán's inequality for these classical polynomials is established by using differential identities that are characteristic of classical polynomials. This seems to be the only case that lends itself to that technique.

Obviously, if

$$
\delta_{n}>\max _{a \leq x \leq b} \frac{P_{n+1}(x) P_{n-1}(x)}{P_{n}^{2}(x)},
$$

then

$$
\delta_{n} P_{n}^{2}(x)-P_{n+1}(x) P_{n-1}(x)>0, \quad a \leq x \leq b .
$$

Inequalities of the form (1.7) will be called weak Turán inequalities to distinguish them from (1.3) which will simply be referred to as Turán inequalities.

2. A weak Turán inequality for sieved ultraspherical polynomials of the second kind. The sieved ultraspherical polynomials were discovered by Al-Salam et al. [1]. Ismail [2, 4] investigated them at great length. If $k \geq 2$ is an integer, then the sieved ultraspherical polynomials of the second kind, $B_{n}^{\lambda}(x ; k)$, satisfy

$$
\begin{gathered}
B_{n+1}^{\lambda}(x ; k)=2 x B_{n}^{\lambda}(x ; k)-B_{n-1}^{\lambda}(x ; k), \quad n+1 \neq m k, \\
m B_{m k}^{\lambda}(x ; k)=2 x(m+\lambda) B_{m k-1}^{\lambda}(x ; k)-(m+2 \lambda) B_{m k-2}^{\lambda}(x ; k), \quad m>0,
\end{gathered}
$$

where $B_{0}^{\lambda}(x ; k)=1, B_{1}^{\lambda}(x ; k)=2 x, k \geq 2$.

Charris and Ismail [4] proved the following remarkable formula that is critical in deriving a weak Turán inequality for these polynomials,

$$
\begin{aligned}
B_{m k+j}^{\lambda}(x ; k)=U_{j}(x) C_{m}^{\lambda+1}\left(T_{k}(x)\right)+U_{k-j-2}(x) C_{m-1}^{\lambda+1}\left(T_{k}(x)\right), & \\
& j=0,1, \ldots, k-1 ; m=0,1,2, \ldots .
\end{aligned}
$$

In (2.2), $U_{j}(x)$ and $T_{k}(x)$ are the Chebychev polynomials of the second kind and the first kind, respectively, and $C_{m}^{\lambda}(x)$ are the ultraspherical polynomials. We recall that if $x=\cos \theta$, then

$$
T_{k}(x)=\cos k \theta, \quad U_{j}(x)=\frac{\sin (j+1) \theta}{\sin \theta} .
$$

We note that $U_{-1}(x)=0$ and $U_{-j}(x)=-(\sin (j-1) \theta / \sin \theta)=-U_{j-2}(x), j \neq 1$. First, we remark that the sieved ultraspherical polynomials do not in general satisfy $\Delta_{n}(x)=$ $\left(B_{n}(x ; k)\right)^{2}-B_{n+1}(x ; k) B_{n-1}(x ; k)>0$. The inequality fails when $n=m k+k-1$ 
because a lengthy calculation, which we omit, shows that

$$
\Delta_{m k+k-1}(1)=\frac{(2 \lambda+2)_{m-1}(2 \lambda+1)_{m+1}(2 \lambda-2 k+1)}{(m+1) ! m !} .
$$

This last quantity may become negative depending on $k$ and $\lambda$. Note that $(a)_{n}$ is defined by $(a)_{n}=a(a+1) \cdots(a+n-1)$.

THEOREM 2.1. Let $\Delta_{n}=\delta_{n}\left[B_{n}^{\lambda}(x ; k)\right]^{2}-B_{n+1}^{\lambda}(x ; k) B_{n-1}^{\lambda}(x ; k)$, where $k \geq 2$ and $\delta_{n}=1$ if $n=m k+j, j=0,1, \ldots, k-2, m=0,1, \ldots$, and $\delta_{m k+k-1}=(m+\lambda+1) /(m+1)$. Then $\Delta_{n}>0$ for $-1 \leq x \leq 1, n=0,1, \ldots$.

Proof. First, we prove that $\Delta_{n}>0$ for $n=m k+j, j=0,1, \ldots, k-2$. Using elementary trigonometric identities, we can show that

$$
U_{j}^{2}-U_{j-1} U_{j+1}=1, \quad 2 U_{j} U_{k-j-2}-U_{j+1} U_{k-j-1}-U_{j-1} U_{k-j-3}=-2 T_{k} .
$$

Using (2.2), we have

$$
\begin{aligned}
\Delta_{m k+j}(x)= & \left(U_{j} C_{m}^{\lambda+1}\left(T_{k}\right)+U_{k-j-2} C_{m-1}^{\lambda+1}\left(T_{k}\right)\right)^{2}-\left(U_{j+1} C_{m}^{\lambda+1}\left(T_{k}\right)+U_{k-j-3} C_{m-1}^{\lambda+1}\left(T_{k}\right)\right) \\
& \cdot\left(U_{j-1} C_{m}^{\lambda+1}\left(T_{k}\right)+U_{k-j-1} C_{m-1}^{\lambda+1}\left(T_{k}\right)\right) \\
= & \left(U_{j}^{2}-U_{j-1} U_{j+1}\right)\left(C_{m}^{\lambda+1}\left(T_{k}\right)\right)^{2}+\left(U_{k-j-2}^{2}-U_{k-j-3} U_{k-j-1}\right)\left(C_{m-1}^{\lambda+1}\left(T_{k}\right)\right)^{2} \\
& +\left(2 U_{j} U_{k-j-2}-U_{j+1} U_{k-j-1}-U_{j-1} U_{k-j-3}\right) C_{m}^{\lambda+1}\left(T_{k}\right) C_{m-1}^{\lambda+1}\left(T_{k}\right)
\end{aligned}
$$

From (2.5), the above equation yields

$$
\begin{aligned}
\Delta_{m k+j}(x) & =\left(C_{m}^{\lambda+1}\left(T_{k}\right)\right)^{2}-2 T_{k} C_{m}^{\lambda+1}\left(T_{k}\right) C_{m-1}^{\lambda+1}\left(T_{k}\right)+\left(C_{m-1}^{\lambda+1}\left(T_{k}\right)\right)^{2} \\
& =\left(C_{m}^{\lambda+1}\left(T_{k}\right)-T_{k} C_{m-1}^{\lambda+1}\left(T_{k}\right)\right)^{2}+\left(1-T_{k}^{2}\right)\left(C_{m-1}^{\lambda+1}\left(T_{k}\right)\right)^{2} \\
& =\left(C_{m}^{\lambda+1}\left(T_{k}\right)-T_{k} C_{m-1}^{\lambda+1}\left(T_{k}\right)\right)^{2}+\sin ^{2} k \theta\left(C_{m-1}^{\lambda+1}\left(T_{k}\right)\right)^{2}>0 .
\end{aligned}
$$

For the case $n=m k+k-1$, we need the recursion for ultraspherical polynomials

$$
(n+1) C_{n+1}^{\lambda}(x)=2(n+\lambda) x C_{n}^{\lambda}(x)-(n+2 \lambda-1) C_{n-1}^{\lambda}(x), \quad n \geq 1,
$$

where $C_{0}^{\lambda}=1, C_{1}^{\lambda}=2 \lambda x$.

Using elementary trigonometric identities, we obtain

$$
\begin{gathered}
U_{n}=U_{n-2}+2 T_{n}, \\
\left(U_{n-1}\right)^{2}-\left(U_{n-2}\right)^{2}-2 T_{n} U_{n-2}=1 .
\end{gathered}
$$

Using (2.2), we get the following formula when $j=k-1$, writing $\theta=(m+\lambda+1) /(m+1)$ for simplicity,

$$
\begin{aligned}
\Delta_{m k+k-1}(x)= & \theta\left(B_{m k+k-1}^{\lambda}\right)^{2}-B_{m k+k-2}^{\lambda} B_{m k+k}^{\lambda} \\
= & \theta\left(U_{k-1} C_{m}^{\lambda+1}\left(T_{k}\right)\right)^{2}-\left(U_{k-2} C_{m}^{\lambda+1}\left(T_{k}\right)+C_{m-1}^{\lambda+1}\left(T_{k}\right)\right)\left(C_{m+1}^{\lambda+1}\left(T_{k}\right)+U_{k-2} C_{m}^{\lambda+1}\left(T_{k}\right)\right) \\
= & \theta\left(U_{k-1}\right)^{2}\left(C_{m}^{\lambda+1}\left(T_{k}\right)\right)^{2}-U_{k-2} C_{m}^{\lambda+1}\left(T_{k}\right) C_{m+1}^{\lambda+1}\left(T_{k}\right)-\left(U_{k-2}\right)^{2} C_{m}^{\lambda+1}\left(T_{k}\right) \\
& -C_{m-1}^{\lambda+1}\left(T_{k}\right) C_{m+1}^{\lambda+1}\left(T_{k}\right)-U_{k-2} C_{m-1}^{\lambda+1}\left(T_{k}\right) C_{m}^{\lambda+1}\left(T_{k}\right) .
\end{aligned}
$$


From (2.8), the above equation yields

$$
\begin{aligned}
\Delta_{m k+k-1}(x)= & \left\{\theta\left(U_{k-1}\right)^{2}-\left(U_{k-2}\right)^{2}\right\}\left(C_{m}^{\lambda+1}\left(T_{k}\right)\right)^{2}-U_{k-2} C_{m}^{\lambda+1}\left(T_{k}\right) \\
& \cdot\left\{\frac{2(m+\lambda+1)}{m+1} T_{k} C_{m}^{\lambda+1}\left(T_{k}\right)-\frac{m+2 \lambda+1}{m+1} C_{m-1}^{\lambda+1}\left(T_{k}\right)\right\} \\
& -C_{m-1}^{\lambda+1}\left(T_{k}\right) \cdot\left\{\frac{2(m+\lambda+1)}{m+1} T_{k} C_{m}^{\lambda+1}\left(T_{k}\right)-\frac{m+2 \lambda+1}{m+1} C_{m-1}^{\lambda+1}\left(T_{k}\right)\right\} \\
& -U_{k-2} C_{m-1}^{\lambda+1}\left(T_{k}\right) C_{m}^{\lambda+1}\left(T_{k}\right) \\
= & \left\{\theta\left(U_{k-1}\right)^{2}-\left(U_{k-2}\right)^{2}-\frac{2(m+\lambda+1)}{m+1} T_{k} U_{k-2}\right\}\left(C_{m}^{\lambda+1}\left(T_{k}\right)\right)^{2} \\
& +\left(\frac{2 \lambda}{m+1} U_{k-2}-\frac{2(m+\lambda+1)}{m+1} T_{k}\right) C_{m}^{\lambda+1}\left(T_{k}\right) C_{m-1}^{\lambda+1}\left(T_{k}\right) \\
& +\frac{m+2 \lambda+1}{m+1}\left(C_{m-1}^{\lambda+1}\left(T_{k}\right)\right)^{2} .
\end{aligned}
$$

Multiplying by $(m+1)$ on both sides and using (2.10), we now have

$$
\begin{aligned}
(m+1) & \Delta_{m k+k-1}(x) \\
= & \left\{(m+\lambda+1)+\lambda U_{k-2}^{2}\right\}\left(C_{m}^{\lambda+1}\left(T_{k}\right)\right)^{2}+2\left\{\lambda U_{k-2}-(m+\lambda+1) T_{k}\right\} \\
& \cdot C_{m}^{\lambda+1}\left(T_{k}\right) C_{m-1}^{\lambda+1}\left(T_{k}\right)+(m+2 \lambda+1)\left(C_{m-1}^{\lambda+1}\left(T_{k}\right)\right)^{2} \\
= & \left\{(m+\lambda+1)+\lambda U_{k-2}^{2}\right\}\left[C_{m}^{\lambda+1}\left(T_{k}\right)+\frac{\lambda U_{k-2}-(m+\lambda+1) T_{k}}{(m+\lambda+1)+\lambda U_{k-2}^{2}} C_{m-1}^{\lambda+1}\left(T_{k}\right)\right]^{2} \\
& +\left[\left\{(m+\lambda+1)+\lambda U_{k-2}^{2}\right\}(m+2 \lambda+1)-\left\{\lambda U_{k-2}-(m+\lambda+1) T_{k}\right\}^{2}\right]\left(C_{m-1}^{\lambda+1}\left(T_{k}\right)\right)^{2} .
\end{aligned}
$$

To complete the proof, we show that the last coefficient of $\left(C_{m-1}^{\lambda+1}\left(T_{k}\right)\right)^{2}$ must be positive.

We have

$$
\begin{aligned}
\{(m+ & \left.\lambda+1)+\lambda\left(U_{k-2}\right)^{2}\right\}(m+2 \lambda+1)-\left\{\lambda U_{k-2}-(m+\lambda+1) T_{k}\right\}^{2} \\
= & (m+\lambda+1)(m+2 \lambda+1)+\lambda(m+2 \lambda+1)\left(U_{k-2}\right)^{2} \\
& -\lambda^{2}\left(U_{k-2}\right)^{2}+2 \lambda(m+\lambda+1) U_{k-2} T_{k}-(m+\lambda+1)^{2}\left(T_{k}\right)^{2} \\
= & (m+\lambda+1)^{2}\left(1-\left(T_{k}\right)^{2}\right)+\lambda(m+\lambda+1) \\
& +\lambda(m+\lambda+1)\left(U_{k-2}\right)^{2}+2 \lambda(m+\lambda+1) U_{k-2} T_{k} \\
= & (m+\lambda+1)^{2}\left(1-\left(T_{k}\right)^{2}\right)+\lambda(m+\lambda+1)\left\{1+\left(U_{k-2}\right)^{2}+2 U_{k-2} T_{k}\right\} .
\end{aligned}
$$

By using (2.10), the above equation yields

$$
\begin{aligned}
(m+\lambda+1)^{2}\left(1-x^{2}\right)\left(U_{k-1}\right)^{2} & +\lambda(m+\lambda+1)\left(U_{k-1}\right)^{2} \\
& =(m+\lambda+1)\left(U_{k-1}\right)^{2}\left\{(m+\lambda+1)\left(1-x^{2}\right)+\lambda\right\}>0
\end{aligned}
$$

(in view of $1-\left(T_{k}\right)^{2}=\left(1-x^{2}\right)\left(U_{k-1}\right)^{2}$ ). This completes the proof of Theorem 2.1. 
REMARK 2.2. The factor $(m+\lambda+1) /(m+1)$ that appears in the statement of Theorem 2.1 cannot be improved with a smaller number. This is because of the asymptotic relation

$$
\lim _{|x| \rightarrow \infty} \frac{B_{m k+k-2}^{\lambda}(x) B_{m k+k}^{\lambda}(x)}{\left(B_{m k+k-1}^{\lambda}(x)\right)^{2}}=\frac{m+\lambda+1}{m+1} .
$$

3. A further determinant of sieved ultraspherical polynomials. In [3], Bustoz and Savage proved an inequality for ultraspherical polynomials of the form

$$
C_{n}^{\alpha}(x) C_{n+1}^{\beta}(x)-C_{n+1}^{\alpha}(x) C_{n}^{\beta}(x)>0, \quad 0<x<1,0<\beta-\alpha \leq 1 .
$$

This inequality was used to answer a conjecture of Askey and Gasper regarding a trigonometric kernel. The question arises if a similar inequality might hold for sieved ultraspherical polynomials. Here, we prove that the analogous inequality does not hold and we determine the location of sign changes for the corresponding sieved expression. We begin with some lemmas.

LEMmA 3.1. Define $\Delta_{n}(x)=C_{n}^{\lambda}(x) C_{n+1}^{\lambda+1}(x)-C_{n+1}^{\lambda}(x) C_{n}^{\lambda+1}(x)$, where $\left\{C_{n}^{\lambda}(x)\right\}$ are the ultraspherical polynomials. Then

$$
\Delta_{n}(x)=a_{n} x\left[C_{n}^{\lambda}(x)\right]^{2}+b_{n} \Delta_{n-2}(x), \quad n \geq 2,
$$

where $a_{n}=(2(n+\lambda) / \lambda(n+1)), b_{n}=((n+2 \lambda-1)(n+2 \lambda-2) / n(n+1))$.

Proof. Each side of (3.2) is a polynomial of degree $2 n+1$. Thus, identity holds in (3.2) if both sides are equal at $2 n+1$ points. We prove equality at the roots $C_{n}^{\lambda}(x)=$ $0, C_{n+1}^{\lambda}(x)=0$. First, note that (3.2) holds when $x=0$. Thus, we may focus on the nonzero roots of $C_{n}^{\lambda}(x)$ and $C_{n+1}^{\lambda}(x)$. Let $C_{n}^{\lambda}(a)=0$. The left-hand side of (3.2) reduces to $\Delta_{n}(a)=-P_{n+1}^{\lambda}(a) P_{n}^{\lambda+1}(a)$. We show that the right-hand side of (3.2) has the same value. From the recursion (2.8), it follows that

$$
C_{n-1}^{\lambda}(a)=-\frac{(n+1) C_{n+1}^{\lambda}(a)}{n+2 \lambda-1}, \quad C_{n-2}^{\lambda}(a)=-\frac{2(n+\lambda-1)(n+1) a C_{n+1}^{\lambda}(a)}{(n+2 \lambda-2)(n+2 \lambda-1)} .
$$

From the relation

$$
(n+\lambda) C_{n}^{\lambda}(x)=\lambda\left[C_{n}^{\lambda+1}(x)-C_{n-2}^{\lambda+1}(x)\right],
$$

it follows that

$$
C_{n-2}^{\lambda+1}(a)=C_{n}^{\lambda+1}(a),
$$

and from the recursion (2.8) we get

$$
C_{n-1}^{\lambda+1}(a)=\frac{C_{n}^{\lambda+1}(a)}{a} .
$$

Then it follows that the right-hand side of (3.2) reduces to $-C_{n+1}^{\lambda}(a) C_{n}^{\lambda+1}(a)$. Thus, (3.2) holds at the $n$ roots of $C_{n}^{\lambda}(x)$. In a very similar fashion, it can be shown that (3.2) holds at the $n+1$ roots of $C_{n+1}^{\lambda}(x)$. This proves the lemma.

By iterating (3.2) we get the following corollary. 
COROLLARY 3.2.

$$
\Delta_{n}(x)=a_{n} x\left[C_{n}^{\lambda}(x)\right]^{2}+x \sum_{k=1}^{[n / 2]} d_{k}\left[C_{n-2 k}^{\lambda}(x)\right]^{2}, \quad \lambda>0, n \geq 2,
$$

where $a_{n}=(2(n+\lambda) / \lambda(n+1))$,

$$
d_{k}=a_{n-2 k} \prod_{j=0}^{k-1} b_{n-2 j}, \quad b_{n}=\frac{(n+2 \lambda-1)(n+2 \lambda-2)}{n(n+1)} .
$$

LEMMA 3.3. Let $\left\{U_{n}(x)\right\}$ denote the sequence of Chebychev polynomials of the second kind. Then

$$
U_{j}(x) U_{k-j-1}(x)-U_{j-1}(x) U_{k-j-2}(x)=U_{k-1}(x) .
$$

Proof. We write $x=\cos \theta$ so that $U_{n}(x)=(\sin (n+1) \theta / \sin \theta)$ and apply trigonometric identities.

THEOREM 3.4. Let $D_{m k+j}(x)=B_{m k+j-1}^{\lambda}(x) B_{m k+j}^{\lambda+1}(x)-B_{m k+j}^{\lambda}(x) B_{m k+j-1}^{\lambda+1}(x), k \geq 2$, $m \geq 3$, and $j=0,1, \ldots, k-1$. If $\lambda>0$, then

$$
D_{m k+j}(x)=U_{k-1}(x) T_{k}(x)\left\{a_{m-1}\left[c_{m-1}^{\lambda+1}\left(T_{k}(x)\right)\right]^{2}+\sum_{k=1}^{[(m-1) / 2]} \gamma_{k}\left[C_{m-1-2 k}^{\lambda+1}\left(T_{k}(x)\right)\right]^{2}\right\},
$$

where $T_{k}(x), U_{k-1}(x)$ are Chebychev polynomials of the first and second kind,

$$
a_{m-1}=\frac{2(m+\lambda)}{m(\lambda+1)}, \quad \gamma_{k}=a_{m-2 k-1} \prod_{j=0}^{k-1} b_{m-2 j-1}, \quad b_{m-1}=\frac{(m+2 \lambda)(m+2 \lambda-1)}{m(m-1)} .
$$

Proof. From Lemma 3.3 after reducing, we get

$$
D_{m k+j}(x)=U_{k-1}(x)\left[C_{m-1}^{\lambda+1}\left(T_{k}(x)\right) C_{m}^{\lambda+2}\left(T_{k}(x)\right)-C_{m}^{\lambda+1}\left(T_{k}(x)\right) C_{m-1}^{\lambda+2}\left(T_{k}(x)\right)\right] .
$$

Now, apply Corollary 3.2. This gives the statement of the theorem.

It is clear from Theorem 3.4 that the determinants $D_{m k+j}(x)$ change sign at the roots of the Chebychev polynomials $T_{k}(x), U_{k-1}(x)$.

\section{REFERENCES}

[1] W. Al-Salam, W. R. Allaway, and R. Askey, Sieved ultraspherical polynomials, Trans. Amer. Math. Soc. 284 (1984), no. 1, 39-55. MR 85j:33005. Zbl 547.33005.

[2] J. Bustoz, M. E. H. Ismail, and J. Wimp, On sieved orthogonal polynomials. VI. Differential equations, Differential Integral Equations 3 (1990), no. 4, 757-766. MR 91a:33005. Zbl 719.33005 .

[3] J. Bustoz and N. Savage, Inequalities for ultraspherical and Laguerre polynomials, SIAM J. Math. Anal. 10 (1979), no. 5, 902-912. MR 80k:33009. Zbl 412.33010.

[4] J. Charris and M. E. H. Ismail, On sieved orthogonal polynomials. II. Random walk polynomials, Canad. J. Math. 38 (1986), no. 2, 397-415. MR 87j:33014a. Zbl 585.33009. 
[5] G. Gasper, On the extension of Turán's inequality to Jacobi polynomials, Duke Math. J. 38 (1971), 415-428. MR 43\#2258. Zbl 214.31401.

[6] __ An inequality of Turán type for Jacobi polynomials, Proc. Amer. Math. Soc. 32 (1972), 435-439. MR 44\#7013. Zbl 237.33009.

[7] O. Szász, Identities and inequalities concerning orthogonal polynomials and Bessel functions, J. Analyse Math. 1 (1951), 116-134. MR 13,232f. Zbl 045.34403.

[8] G. Szegö, On an inequality of P. Turán concerning Legendre polynomials, Bull. Amer. Math. Soc. 54 (1948), 401-405. MR 9,429d. Zbl 032.27502.

[9] P. Turán, On the zeros of the polynomials of Legendre, Časopis Pěst. Mat. Fys. 75 (1950), 113-122. MR 12,824g. Zbl 040.32303.

Joaquin Bustoz: Department of Mathematics, Arizona State University, Tempe, AZ 85287, USA

E-mail address: bustoz@asu.edu

I. S. Pyung: Korean NAVAl ACAdemy, Chin-Hae, Kyung-NAm, 645-797, KoreA 


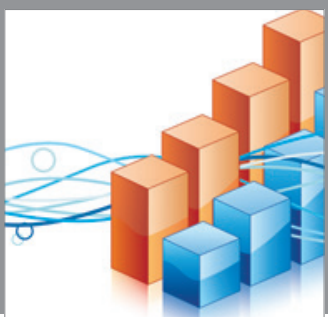

Advances in

Operations Research

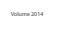

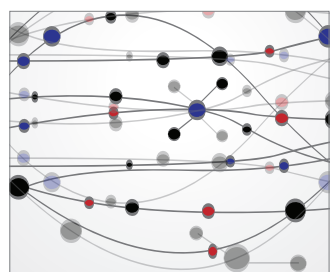

\section{The Scientific} World Journal
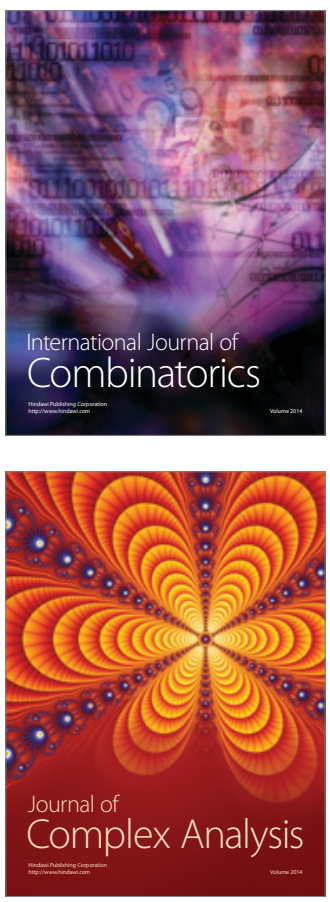

International Journal of

Mathematics and

Mathematical

Sciences
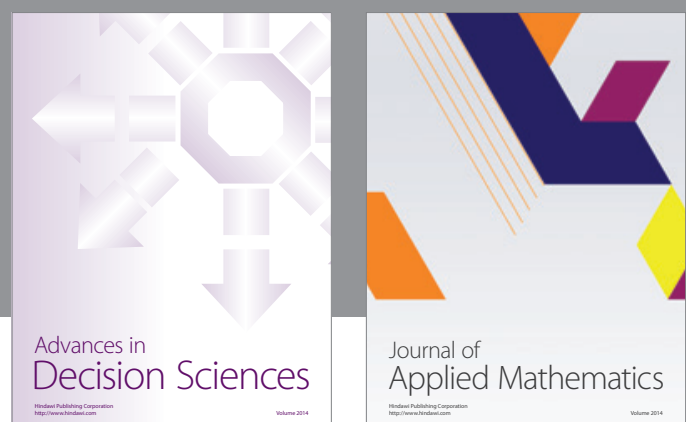

Journal of

Applied Mathematics
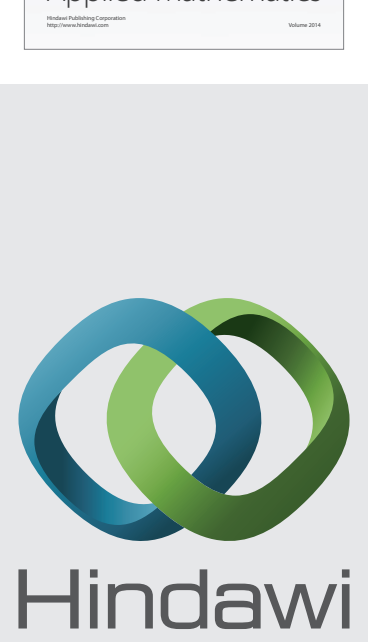

Submit your manuscripts at http://www.hindawi.com
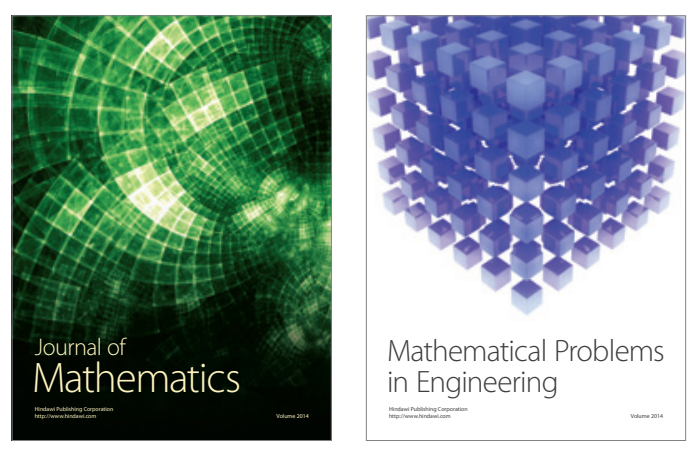

Mathematical Problems in Engineering
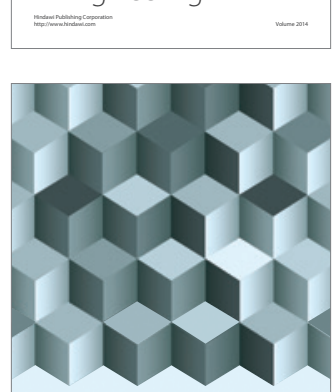

Journal of

Function Spaces
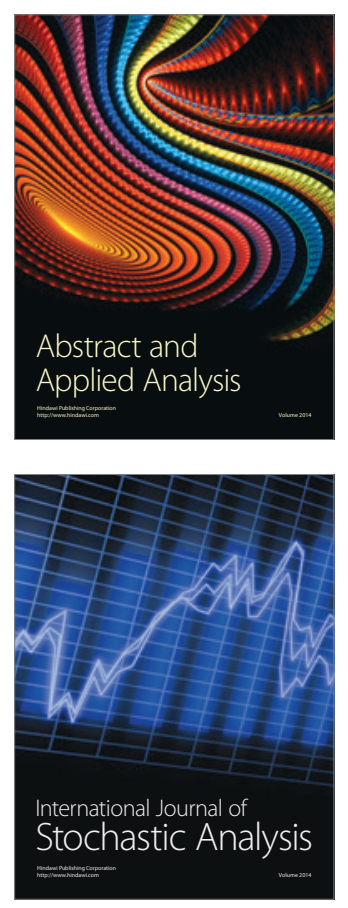

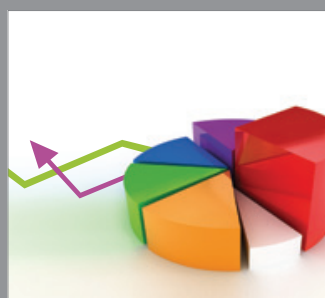

ournal of

Probability and Statistics

Promensencen
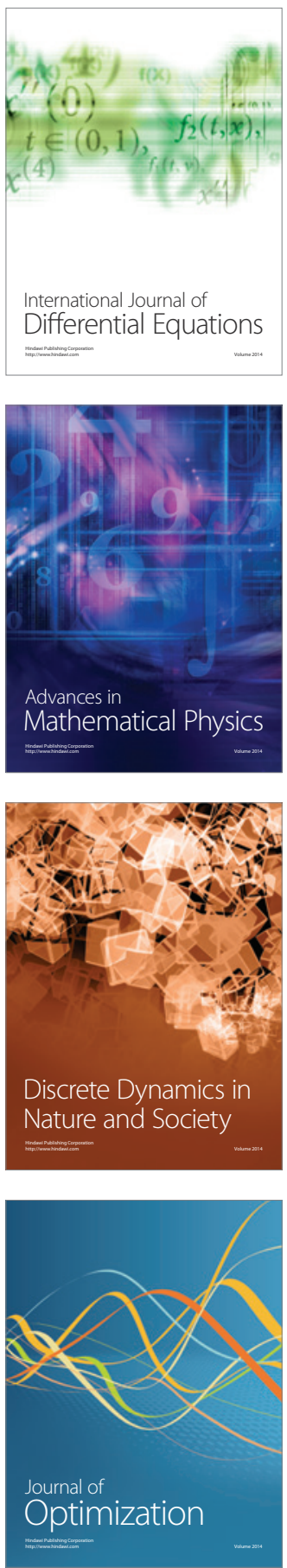